\title{
THC reduces the anticipatory nucleus accumbens response to reward in subjects with a nicotine addiction
}

\author{
JM Jansma ${ }^{1,7}$, HH van Hell ${ }^{1,7}$, LJMJ Vanderschuren ${ }^{2,3}$, MG Bossong ${ }^{1,4}$, G Jager $^{1,5}$, RS Kahn $^{6}$ and NF Ramsey ${ }^{1}$
}

Recent evidence has implicated the endocannabinoid (eCB) system in nicotine addiction. The eCB system also has an important role in reward mechanisms, and nicotine addiction has been associated with aberrant reward processing. Motivated by this evidence, we tested the hypothesis that $\mathrm{ECB}$ modulation of reward processing is altered in subjects with a nicotine addiction (NAD). For this purpose, we compared reward-related activity in NAD with healthy controls (HC) in a pharmacological magnetic resonance imaging (MRI) study using $\Delta^{9}$-tetrahydrocannabinol (THC) administration to challenge the eCB system. Eleven HC and 10 NAD participated in a 3-T functional MRI (fMRI) study with a double-blind, cross-over, placebo-controlled design, using a Monetary Incentive Delay (MID) paradigm with three reward levels. Reward activity in the nucleus accumbens (NAcc) and caudate putamen during anticipation and feedback of reward was compared after THC and placebo. fMRI results indicated a significant reduction of reward anticipation activity in the NACc in NAD after THC administration, which was not present in HC. This is indicated by a significant group by drug by reward interaction. Our data show that THC significantly reduces the NAcc response to monetary reward anticipation in NAD. These results suggest that nicotine addiction is associated with altered eCB modulation of reward processing in the NAcc. This study adds important human data to existing evidence implicating the eCB system in nicotine addiction.

Translational Psychiatry (2013) 3, e234; doi:10.1038/tp.2013.6; published online 26 February 2013

\section{Introduction}

Recent estimates of the World Health Organization have shown that there are about 1.25 billion smokers worldwide and that five million deaths occur each year as a direct result of tobacco use. ${ }^{1}$ This makes tobacco use one of the most important causes of preventable death worldwide.

There is increasing evidence that the endocannabinoid $(\mathrm{eCB})$ system modulates the addictive properties of nicotine, the main addictive ingredient of tobacco, as well as several other drugs (see for review, refs. (2-7)). Animal studies have shown that blocking the eCB receptor $\mathrm{CB} 1$ with the antagonist rimonabant reduces self-administration of nicotine $e^{4,8}$ and relapse to nicotine seeking (as well as other drugs, such as cocaine and ethanol). ${ }^{9}$ Nicotine withdrawal has been shown to be accompanied by fluctuations in the levels of the eCB anandamide (AEA) in several brain structures in rats. ${ }^{10}$ In humans, clinical trials have indicated that rimonabant can facilitate smoking cessation. ${ }^{2,11}$ The eCB system has also been widely implicated in the reward properties of non-drugs, ${ }^{12,13}$ which has recently been supported by human neuroimaging studies. ${ }^{14-17}$ Preclinical studies have, for instance, shown that $\mathrm{CB} 1$ receptor agonists can increase food intake, ${ }^{18,19}$ whereas the CB1 antagonist rimonabant has been demonstrated to reduce obesity ${ }^{11}$ and reduce striatal brain activity during reward processing. ${ }^{17}$ In addition, there is evidence of deficient reward processing in nicotine addiction. ${ }^{20-23}$

Taken together, these findings suggest the possibility that nicotine addiction may be associated with an altered interaction between the eCB system and reward processing. ${ }^{13}$ This paper presents the results of the first human neuroimaging study that tested the hypothesis that nicotine addiction is associated with abnormal involvement of the eCB system in reward processing. The study builds upon a previous study, in which a similar method was used to examine the involvement of the eCB system in reward processing in a healthy population. ${ }^{16}$ As in the previous study, we performed a double-blind, cross-over, placebo-controlled pharmacological functional magnetic resonance imaging (fMRI) study probing the brain reward system in which the eCB system was challenged with $\Delta^{9}$-tetrahydrocannabinol (THC). Importantly, in the current study, we compare the effects of THC on

\footnotetext{
${ }^{1}$ Department of Neurology and Neurosurgery, Rudolf Magnus Institute of Neuroscience, University Medical Center Utrecht, Utrecht, The Netherlands; ${ }^{2}$ Department of Neuroscience and Pharmacology, Rudolf Magnus Institute of Neuroscience, University Medical Center Utrecht, Utrecht, The Netherlands; ${ }^{3}$ Department of Animals in Science and Society, Division of Behavioural Neuroscience, Faculty of Veterinary Medicine, Utrecht University, Utrecht, The Netherlands; ${ }^{4}$ Department of Psychosis Studies, King's College, Institute of Psychiatry, London, UK; ${ }^{5}$ Division of Human Nutrition, Wageningen University, Wageningen, The Netherlands and ${ }^{6}$ Department of Psychiatry, Rudolf Magnus Institute of Neuroscience, University Medical Center Utrecht, Utrecht, The Netherlands

Correspondence: Professor NF Ramsey, Department of Neurology and Neurosurgery, Rudolf Magnus Institute of Neuroscience, University Medical Center Utrecht, Heidelberglaan 100, Room G.03.124, Utrecht 3584 CX, The Netherlands.

E-mail: N.Ramsey@umcutrecht.nl

${ }^{7}$ The first two authors contributed equally to this work.

Keywords: endocannabinoid system; functional MRI; nicotine addiction; nucleus accumbens; reward; THC

Received 10 July 2012; revised 1 December 2012; accepted 3 January 2013
} 
reward processing in a sample of subjects with a nicotine addiction (NAD) with a new sample of healthy controls (HC). Reward processing was assessed using a task based on the Monetary Incentive Delay (MID) task. ${ }^{24}$ The MID is an established reward paradigm that provides the possibility of separate measures of brain activity related to anticipation of reward as well as to notification that the reward has been won. Previous studies using this paradigm have indicated that anticipation of a reward activates the ventral striatum, especially in the nucleus accumbens (NAcc). ${ }^{24}$

Image analyses were focused on the NAcc and caudate putamen (CPU), areas that have been implicated in addiction $^{25-27}$ as well as reward processing. ${ }^{14,28}$ Based on our hypothesis that nicotine addiction is associated with abnormal involvement of the eCB system in reward processing, we expect that $\mathrm{THC}$ administration has a stronger effect on the response of the brain to rewarding stimuli in NAD than in $\mathrm{HC}$. We postulate that $\mathrm{THC}$ should enhance the brain response to natural rewards in $\mathrm{HC}$, which is in line with previous publications. ${ }^{13,17}$ We further expect the enhanced sensitivity to THC in NAD to lead to a maximized level of activity in the reward system, resulting in a significantly diminished additional response to natural rewards.

\section{Methods and materials}

This study is part of the Pharmacological Imaging of the Cannabinoid System (PhICS) study. Methods of the entire study are reported in detail in a methodological paper. ${ }^{29}$ The study is registered in both the EudraCT database (2007004247-30) and the Dutch Trial Register (NTR1787). Although there are previous related publications from the same project, none of the data presented in this manuscript have been used in other publications.

Subjects. Fifteen subjects with a nicotine addiction and 17 healthy male subjects participated in a randomized placebocontrolled cross-over pharmacological MRI study with THC administration. ${ }^{29}$ All volunteers gave written informed consent before entry into the study and were paid 250 euro for participation. The study was approved by the Ethical Committee of the University Medical Center Utrecht in accordance with the Declaration of Helsinki 2008.
All subjects were required by the Ethical Committee to be occasional cannabis users (at least four times a year, at most once a week) who never had negative experiences after cannabis use (for instance, a bad trip or cannabis-induced psychosis), in order to minimize the risk for adverse events. Subjects were excluded if they or their first-degree relatives were diagnosed with a psychiatric disorder, as assessed using the Mini International Neuropsychiatric Interview for DSM-IV clinical disorders. ${ }^{30}$ Subjects included in the $\mathrm{HC}$ group did not score on the nicotine addiction questionnaire FTND (Fagerstrom Test for Nicotine Dependence) ${ }^{31}$ and smoked $<0.5$ cigarettes per day. Subjects included in the nicotine addiction group had a minimal score of 3 on the FTND and smoked at least 10 cigarettes per day. Other inclusion and exclusion criteria are described in detail elsewhere. ${ }^{29}$

Two $\mathrm{HC}$ were excluded due to feelings of anxiety during one of the scanning sessions, two $\mathrm{HC}$ due to movement artefacts and one due to lack of elevated plasma levels of THC. Two NAD were excluded due to movement artefacts, one due to feelings of anxiety during one of the scanning sessions and one due to high blood pressure during the first test day. Two subjects were excluded as they did not fulfill either the inclusion criteria for $\mathrm{HC}$ or for NAD with regard to nicotine addiction as applied to the experimental groups for this study. Results are therefore reported on $11 \mathrm{HC}$ (mean age 21.2 years) and 10 NAD (mean age 25.6 years). Subject characteristics are presented in detail in Table 1. Subject demographic characteristics did not show significant group differences, except as by design for nicotine use last year $(t=11.1, P<0.001)$ as well as the score on the FTND $(t=13.5 ; P \leqslant 0.001)$.

Procedure. At a training session, subjects practiced the procedure of drug administration and participants were familiarized with the scanning procedure in a 'mock scanner' (a non-working replica of a real scanner) to reduce stress effects on the following test days. The actual study consisted of 2 test days, separated by at least 2 weeks to allow for complete clearance of drugs. A standard breakfast or lunch was provided at the beginning of each test day, to ensure equal states of metabolism on both of the test days. Subjects were instructed not to use cannabis for at least 2 weeks before the first test day. Compliance was tested by means of

Table 1 Results and statistical test results for demographic characteristics

\begin{tabular}{|c|c|c|c|c|c|c|}
\hline \multirow[t]{2}{*}{ Category } & \multicolumn{2}{|c|}{$H C$} & \multicolumn{2}{|l|}{$N A D$} & \multicolumn{2}{|c|}{ Effect of group } \\
\hline & Mean ( \pm s.e.m.) & Range & Mean ( \pm s.e.m.) & Range & $t(19)$ & $P$ \\
\hline Age (years) & $21.2 \pm 0.8$ & $18-26$ & $25.6 \pm 2.1$ & $20-40$ & 1.96 & 0.07 \\
\hline IQ & $105.0 \pm 1.5$ & $98-113$ & $107.0 \pm 1.6$ & $98-114$ & 0.91 & 0.37 \\
\hline Cigarettes/day & $0.06 \pm 0.04$ & $0.0-0.4$ & $17.2 \pm 1.5$ & $10-29$ & 11.09 & $<0.001$ \\
\hline FTND & $0.0 \pm 0.0$ & $0-0$ & $4.3 \pm 0.3$ & $3-6$ & 13.50 & $<0.001$ \\
\hline Alcohol units/week & $13.2 \pm 2.4$ & $3-30$ & $14.4 \pm 2.9$ & $2-30$ & 0.33 & 0.75 \\
\hline Cannabis use last year (no. of occasions) & $22.6 \pm 3.6$ & $4-52$ & $23.5 \pm 5.8$ & $5-52$ & 0.13 & 0.90 \\
\hline Illicit drug lifetime (no. of occasions) & $0.73 \pm 0.5$ & $0-6$ & $2.4 \pm 1.3$ & $0-13$ & 1.21 & 0.24 \\
\hline Peak plasma THC level $\left(\mathrm{ng} \mathrm{ml}^{-1}\right)$ & $82.8 \pm 8.2$ & $30-133$ & $81.8 \pm 12.3$ & $27-178$ & 0.08 & 0.94 \\
\hline
\end{tabular}

Abbreviations: FTND, Fagerstrom Test for Nicotine Dependence; HC, healthy controls; NAD, subjects with a nicotine addiction; IQ, Intelligent Quotient; THC, $\Delta^{9}$-tetrahydrocannabinol.

Bold typeface and gray background: $P<0.05$; bold typeface: $P<0.1$. 
a urine sample at the beginning of each test day. Venous blood samples were collected to determine plasma concentrations of THC and its two most important metabolites, 11-OH-THC and 11-nor-9-carboxy-THC. Blood samples were processed according to methods published elsewhere. ${ }^{29}$ Subjective effects were measured using a Visual Analog Scale (VAS) before and after each task, on a scale from -100 to $100 .^{32,33}$

Drug administration. THC or placebo was administered at four time points by inhalation ('breathing') using a Volcano vaporizer ${ }^{29,34}$ (Storz and Bickel $\mathrm{GmbH}$, Tuttlingen, Germany). Five minutes before administration, THC was vaporized at a temperature of $225^{\circ} \mathrm{C}$ into an opaque polythene bag equipped with a mouthpiece, preventing the loss of THC in between inhalations. Subjects inhaled the volume of this bag in 2-3 min, holding their breath for $10 \mathrm{~s}$ after each inhalation. They were not allowed to speak during the inhalation process, which was practiced at screening using placebo. Vehicle (ethanol only) was used as a placebo. The first dose consisted of $6 \mathrm{mg}$ THC or placebo. To maintain equal levels of intoxication effects throughout the experiment, upload dosages of $1 \mathrm{mg}$ were used, $30 \mathrm{~min}$ apart, in between scan sessions of different paradigms.

Task. To activate reward circuitry, an adapted version of the 'MID' task as developed by Knutson et al. ${ }^{24}$ was used. In this task, subjects are required to press a button as fast as possible on seeing a target stimulus. Depending on the cue that precedes the target stimulus, subjects can win or lose a certain amount of money. After each trial in each condition, except neutral, subjects are given visual feedback, about the amount won or lost in that trial, as well as the total amount won (see Figure 1). Brain activity of both anticipation and feedback of reward and loss can be assessed with this task.

Our MID task had four conditions: neutral, reward (10 cents ('small') or 5 euro ('large')) or a loss (5 euro). There were 16 trials for the neutral and reward conditions, and 12 for the loss condition. The function of the loss trials was to prevent adaptation to rewards, and they were not meant to be included in the analysis. The reward earned by subjects was dependent on actual task performance.

The reward cue was presented for $0.5 \mathrm{~s}$, while the feedback was presented for $1.0 \mathrm{~s}$. A correct response ('HIT') was defined as a response before the target disappeared. All other responses are considered incorrect ('MISS'). Target presentation time was individually adapted in order to ensure close to $50 \%$ correct responses for each subject, so that all subjects received comparable total rewards in both of the sessions. This was achieved by presenting half of the trials $200 \mathrm{~ms}$ longer and half of the trails 150 ms shorter than the subject's shortest reaction time $(\mathrm{RT})$, based on 10 practice trials. The intervals between cue and target (the anticipation phase or 'ANT'), between target and feedback, as well as the inter-trial interval were varied to allow for event-related fMRI analysis (see Figure 1).

Scanning parameters. Image acquisition was performed on a Philips Achieva 3.0 Tesla MR scanner with a Quasar dual gradient set (Philips Medical Systems, Best, the Netherlands). Functional imaging was performed in a single run of 1182 volumes (11 min and 51 s) using a SENSEPRESTO scan protocol ${ }^{35}$ (scan parameters: TR (repetition time) $22.5 \mathrm{~ms}$; TE (time to echo) $33.2 \mathrm{~ms}$; flip angle $=10^{\circ}$; FOV (field of view) $224 \times 256 \times 160$; matrix $56 \times 64 \times 40$; voxel size $4.0 \mathrm{~mm}$ isotropic; scan time $0.6075 \mathrm{~s}$; 40 slices; sagittal orientation). Before the functional imaging run, a high-resolution T1-weighted whole-brain anatomical scan was performed (scan parameters: TR $9.4 \mathrm{msec}$; TE $4.7 \mathrm{msec}$; flip angle $=8^{\circ}$; FOV $220.8 \times 240 \times 159.6$; matrix $368 \times 400 \times 113$; voxel size $0.6 \mathrm{~mm} \times 0.6 \mathrm{~mm} \times 0.6 \mathrm{~mm}, 266$ slices; sagittal orientation).

Analysis. All statistical tests were performed with the statistical package IBM SPSS Statistics 20 (IBM, New York, NY, USA).

Demographic, subjective and physiological measures. Demographic data was tested for group differences with

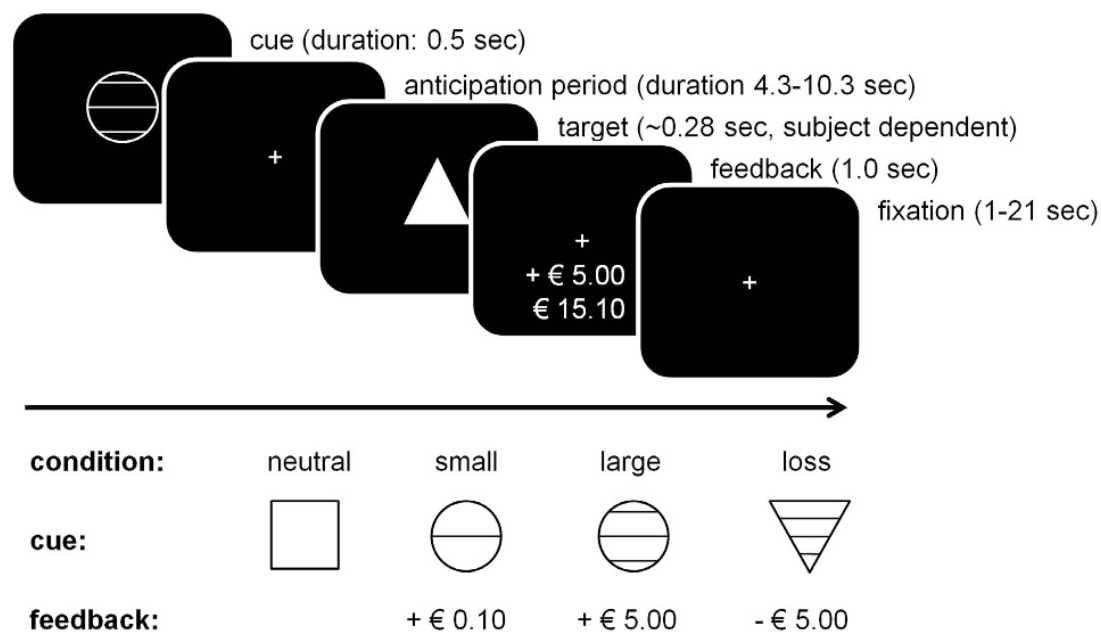

Figure 1 Task paradigm: Each trial started with a fixation cross, followed by a cue signaling a neutral, potential reward (large or small) or potential loss trial. After the cue, a target was presented to which subjects had to respond as fast as possible by pressing a button. Reward and loss trials ended with feedback. The time between cue and target (anticipation phase or 'ANT') was varied between trials (4.3-10.3 s; mean $6.6 \mathrm{~s})$, as well as the inter-trial interval (0-30 s; mean $4.2 \mathrm{~s})$. 
paired sample t-tests. VAS scores were corrected for baseline values and averaged over pre- and post-scan measurement. VAS scores as well as physiological measures were tested for effects of drug in $\mathrm{HC}$ and group by drug effects using analysis of variance (ANOVA).

Task performance. Reward task performance was measured using RTs. A repeated-measures general linear model (GLM) with drug (placebo, THC) and reward (neutral, small and large) as within-subject and group as between-subject factor was performed to tests for effects of THC, reward and nicotine addiction. In addition, several univariate follow-up tests were performed.

fMRI. fMRI data were pre-processed and analyzed using SPM5 (Wellcome Trust Center for Neuroimaging, London, UK). Pre-processing of data consisted of realignment of functional images and co-registration, spatial normalization into standard MNI (Montreal Neurological Institute) space, and spatially smoothing with a Gaussian filter (full-width half-maximum $=8 \mathrm{~mm}$ ). Hypotheses were tested in a region of interest (ROI) analysis in CPU and NAcc. NAcc was defined by a 5-mm sphere around MNI coordinates $-12,14,-8$ (left) and 12, 14, - 8 (right). CPU was defined using the AAL (anatomical automatic labeling) atlas. ${ }^{36}$

A GLM regression analysis was performed in SPM5 using an event-related analysis with factors time-locked to task events, convolved with a canonical hemodynamic response function. The design included a total of 13 regressors. Four regressors modeled anticipation activity for each of the four conditions, using the variable anticipation phase as the expected duration of each event. Eight regressors modeled the feedback activity, one for HITS and one for MISSES for each of the four conditions. Finally, one regressor modeled response activity for all the four conditions.
To test for effects of THC on reward anticipation, we averaged beta values over all voxels included in each $\mathrm{ROI}$, for each condition and each session.

We performed a repeated-measures GLM with ROI (two levels: left and right), drug (two levels: THC and placebo) and reward condition (three levels: neutral, small and large) as within-subject factors and group (HC and NAD) as betweensubject variable. To test for effects of lateralization, we also performed a four-way interaction test with factors: hemisphere (two levels: left and right), drug (two levels: THC and placebo) and reward condition (three levels: neutral, small and large) as within-subject factors and group (HC and NAD) as betweensubject variable. As follow-up analyses to interpret significant interaction effects, we tested for effects of reward by group in the anticipation phase for placebo and THC session separately, as well as for the main effects of reward in both the groups. Finally, we performed tests for main effect of drugs in $\mathrm{HC}$ and NAD, unrelated to reward as well as for drug by group differences in these main effects. All follow-up tests were also performed using GLM (repeated measures).

For the feedback phase, the same set of tests was performed, but now using the contrast between hits and misses for the two conditions that provided feedback ('small' and 'large').

\section{Results}

Subjective and physiological results. Detailed results are presented in Table 1 (peak plasma level) and Table 2. Subjects showed a peak plasma level of 82.8 for $\mathrm{HC}$ and 81.8 for NAD (no significant group difference). VAS scores indicated a significantly increased score for 'feeling high', and 'external perception', as well as a significantly reduced score for 'alertness' and 'contentedness' in HC and NAD. In addition, $\mathrm{HC}$ showed a significantly increased score for 'calmness'. In these categories, only 'calmness' showed a

Table 2 VAS scores

\begin{tabular}{|c|c|c|c|c|c|c|}
\hline \multirow[t]{2}{*}{ Category } & \multicolumn{3}{|c|}{ Placebo (mean \pm s.e.m.) } & \multicolumn{3}{|c|}{$T H C$ (mean \pm s.e.m.) } \\
\hline & \multicolumn{2}{|c|}{$H C$} & $N A D$ & $H C$ & & $N A D$ \\
\hline $\begin{array}{l}\text { Feeling high } \\
\text { Internal perception } \\
\text { External perception } \\
\text { Alertness } \\
\text { Contentedness } \\
\text { Calmness }\end{array}$ & \multicolumn{2}{|c|}{$\begin{array}{r}0.7 \pm 1.0 \\
0.2 \pm 0.2 \\
0.6 \pm 0.6 \\
-4.8 \pm 2.0 \\
-2.9 \pm 2.3 \\
2.2 \pm 3.5\end{array}$} & $\begin{array}{r}0.0 \pm 0.0 \\
0.3 \pm 0.3 \\
0.3 \pm 0.2 \\
-6.2 \pm 2.3 \\
-3.1 \pm 1.6 \\
3.6 \pm 3.6\end{array}$ & $\begin{array}{r}29.5 \pm 9.2 \\
3.0 \pm 2.5 \\
7.3 \pm 2.0 \\
-15.9 \pm 4.0 \\
-10.6 \pm 2.7 \\
-13.8 \pm 4.7\end{array}$ & & $\begin{array}{r}22.0 \pm 6.1 \\
1.1 \pm 0.5 \\
4.7 \pm 1.8 \\
-21.6 \pm 3.2 \\
-10.0 \pm 2.1 \\
0.4 \pm 3.8\end{array}$ \\
\hline \multirow{2}{*}{ Calmness } & \multicolumn{2}{|c|}{ HC (effect of drug) } & \multicolumn{2}{|c|}{$N A D$ (effect of drug) } & \multicolumn{2}{|c|}{ Drug by group } \\
\hline & $t(10)$ & $p$ & $t(9)$ & $P$ & $F(1,19)$ & $p$ \\
\hline $\begin{array}{l}\text { Feeling high } \\
\text { Internal perception } \\
\text { External perception } \\
\text { Alertness } \\
\text { Contentedness } \\
\text { Calmness }\end{array}$ & $\begin{array}{l}3.00 \\
1.10 \\
2.84 \\
2.74 \\
2.85 \\
3.75\end{array}$ & $\begin{array}{l}0.01 \\
0.29 \\
0.01 \\
0.02 \\
0.02 \\
0.004\end{array}$ & $\begin{array}{l}3.66 \\
1.35 \\
2.53 \\
6.48 \\
3.11 \\
0.80\end{array}$ & $\begin{array}{c}\mathbf{0 . 0 0 5} \\
0.21 \\
0.03 \\
<0.001 \\
0.01 \\
0.44\end{array}$ & $\begin{array}{l}0.32 \\
0.52 \\
0.58 \\
0.83 \\
0.06 \\
4.81\end{array}$ & $\begin{array}{l}0.58 \\
0.48 \\
0.46 \\
0.37 \\
0.81 \\
\mathbf{0 . 0 4}\end{array}$ \\
\hline
\end{tabular}

Abbreviations: HC, healthy controls; NAD, subjects with a nicotine addiction; THC, $\Delta^{9}$-tetrahydrocannabinol; VAS, Visual Analog Scale.

Results and statistical test results for subjective measurements, as measured with the VAS, averaged over the pre- and post-scan measurement (scale minimum: - 100, maximum: 100, bold typeface and gray background: $P<0.05$; bold typeface: $P<0.1$ ). 
group by drug interaction effect, indicating that while $\mathrm{THC}$ significantly reduced calmness in $\mathrm{HC}$, there was no effect in NAD.

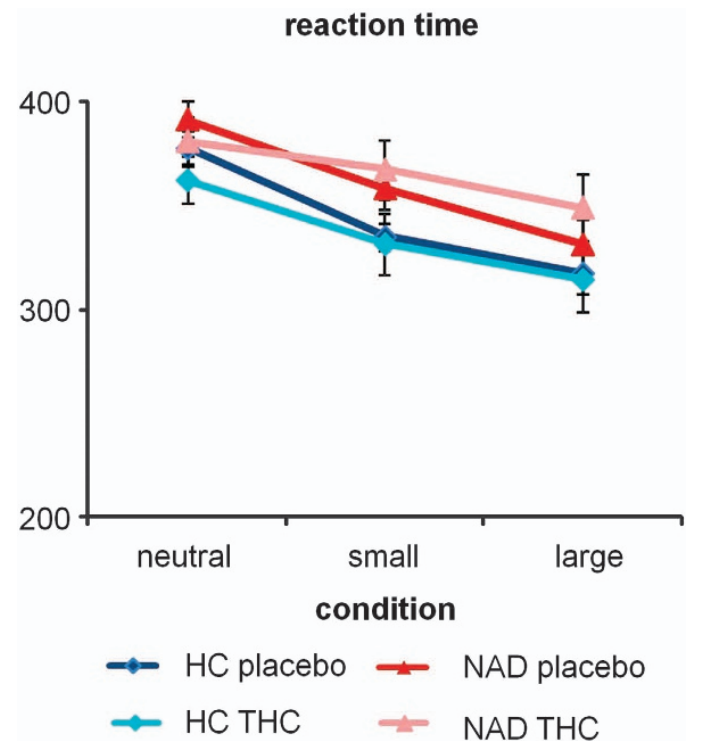

Figure 2 Reaction times (ms) for the conditions with no reward ('neutral'), a 0.10 euro ('small') or a 5 euro reward ('large'); (blue lines, squares: healthy controls $(\mathrm{HC})$; red lines, triangles: subjects with a nicotine addiction (NAD); dark: placebo; light: $\Delta^{9}$-tetrahydrocannabinol (THC)).
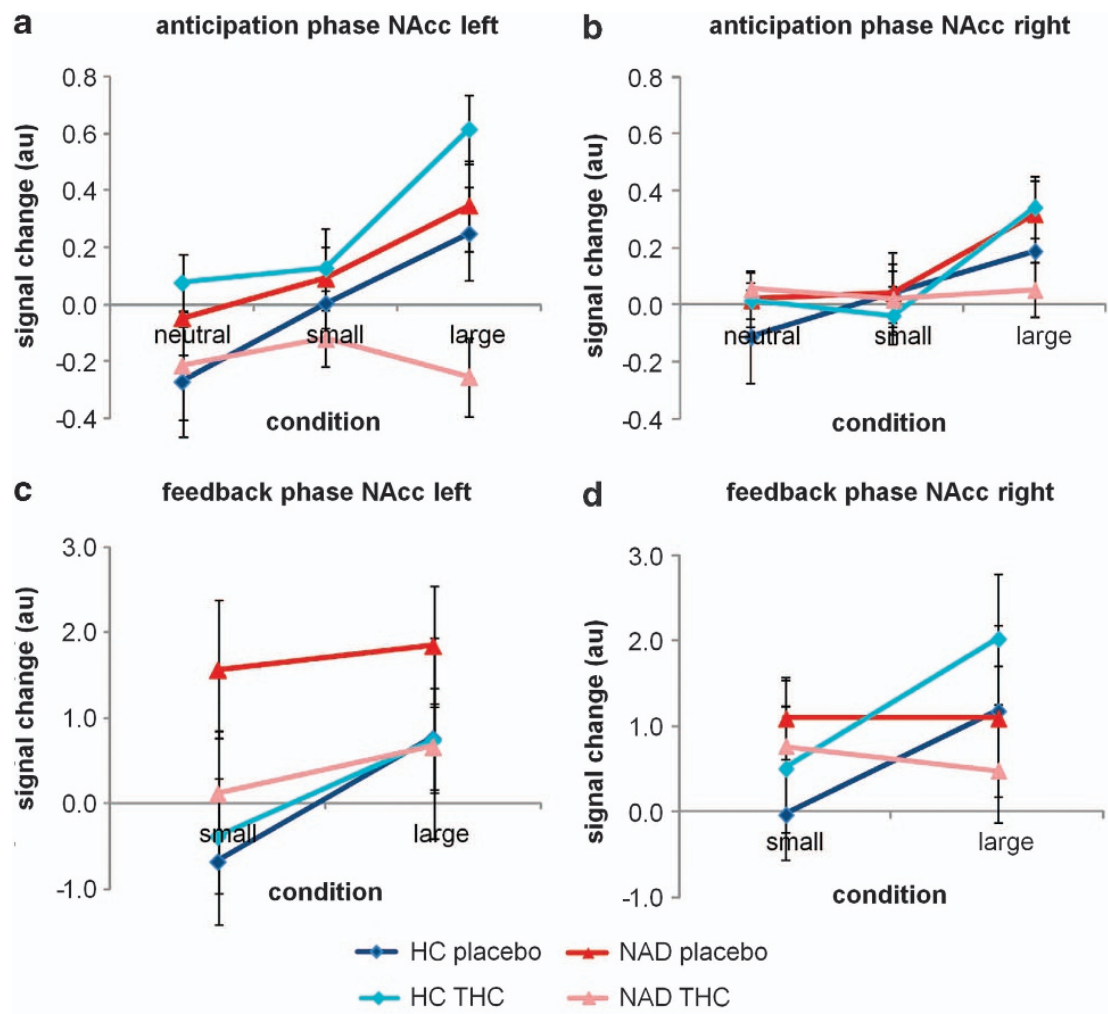

Figure 3 Above: reward anticipation activity in the (a) left and (b) right nucleus accumbens (NAcc) for the conditions with no reward ('neutral'), a 0.10 euro ('small') or a 5 euro reward ('large'); (blue: healthy controls (HC); red: subjects with a nicotine addiction (NAD); dark: placebo, light: $\Delta^{9}$-tetrahydrocannabinol (THC)). HC showed a significant increase in response with increasing reward. Over hemispheres, we found a significant reduction in NAcc activity in NAD after THC administration. Below: reward feedback activity in the (c) left and (d) right NAcc for the feedback conditions with a reward ('small' or large); (blue: HC; red: subjects with a nicotine addiction; dark: placebo; light: THC). au, arbitrary units. 
Table 3 Statistical tests for fMRI measurements

\begin{tabular}{|c|c|c|c|c|c|c|}
\hline \multirow[t]{2}{*}{ Nucleus accumbens } & \multicolumn{3}{|c|}{ Anticipation phase } & \multicolumn{3}{|c|}{ Feedback phase } \\
\hline & $F$ & $P$ & $(d f)$ & $F$ & $P$ & $(d f)$ \\
\hline \multicolumn{7}{|l|}{ Hypothesis tests } \\
\hline Drug by reward by group & 3.73 & 0.04 & $(2,18)$ & 0.01 & 0.99 & $(1,19)$ \\
\hline Hemisphere by drug by reward by group & 0.11 & 0.90 & $(2,18)$ & 0.13 & 0.88 & $(1,19)$ \\
\hline \multicolumn{7}{|l|}{ Follow-up tests } \\
\hline Reward by group (placebo) & 0.35 & 0.71 & $(2,18)$ & 0.85 & 0.37 & $(1,19)$ \\
\hline Reward by group (THC) & 7.64 & $<0.001$ & $(2,18)$ & 1.02 & 0.33 & $(1,19)$ \\
\hline $\mathrm{HC}$ (main effect of reward) & 4.91 & 0.04 & $(2,9)$ & 4.38 & 0.06 & $(1,10)$ \\
\hline NAD (main effect of reward) & 1.36 & 0.31 & $(2,8)$ & 0.05 & 0.83 & $(1,9)$ \\
\hline \multicolumn{7}{|l|}{ Non-reward tests } \\
\hline $\mathrm{HC}$ (main effect of drug) & 1.83 & 0.21 & $(1,10)$ & 0.26 & 0.62 & $(1,10)$ \\
\hline NAD (main effect drug) & 2.15 & 0.18 & $(1,9)$ & 4.05 & 0.08 & $(1,9)$ \\
\hline Drug by group & 3.99 & 0.06 & $(1,19)$ & 1.91 & 0.18 & $(1,19)$ \\
\hline Caudate putamen & $F$ & $p$ & $(d f)$ & $F$ & $p$ & $(d f)$ \\
\hline \multicolumn{7}{|l|}{ Hypothesis tests } \\
\hline Drug by reward by group & 2.50 & 0.11 & $(2,18)$ & 0.07 & 0.80 & $(1,19)$ \\
\hline Hemisphere by drug by reward by group & 1.41 & 0.27 & $(2,18)$ & 0.34 & 0.57 & $(1,19)$ \\
\hline \multicolumn{7}{|l|}{ Follow-up tests } \\
\hline Reward by group (placebo) & 1.89 & 0.18 & $(2,18)$ & 1.81 & 0.19 & $(1,19)$ \\
\hline Reward by group (THC) & 4.36 & 0.03 & $(2,18)$ & 1.16 & 0.30 & $(1,19)$ \\
\hline $\mathrm{HC}$ (main effect of reward) & 11.56 & $<0.001$ & $(2,9)$ & 6.76 & 0.03 & $(1,10)$ \\
\hline NAD (main effect of reward) & 1.03 & 0.40 & $(2,8)$ & 0.01 & 1.00 & $(1,9)$ \\
\hline \multicolumn{7}{|l|}{ Non-reward tests } \\
\hline $\mathrm{HC}$ (main effect of drug) & 0.30 & 0.60 & $(1,10)$ & 0.94 & 0.36 & $(1,10)$ \\
\hline NAD (main effect of drug) & 4.74 & 0.06 & $(1,9)$ & 6.37 & 0.03 & $(1,9)$ \\
\hline Drug by group & 0.85 & 0.37 & $(1,19)$ & 0.45 & 0.51 & $(1,19)$ \\
\hline
\end{tabular}

Abbreviations: df, degrees of freedom; fMRI, functional magnetic resonance imaging; HC, healthy controls; NAD, subjects with a nicotine addiction; THC, $\Delta^{9}$ tetrahydrocannabinol.

Statistical tests for fMRI measurements in nucleus accumbens and caudate putamen (general linear model, repeated measures; bold typeface and gray background: $P<0.05$ bold typeface: $P<0.1$.

shown in Table 3. An illustration of the activity is presented in Figure 5.

Anticipation phase. First, we discuss reward-related effects that are associated with our main hypothesis. For the NAcc, we found that reward increased brain activity in NAcc $(F(2,9)=4.91 ; P=0.04)$, while in NAD there was no significant increase of brain activity with increasing reward $(\mathrm{F}(2,8)=1.36 ; P=0.31)$. After placebo, we found no difference in reward-related activity in NAcc between $\mathrm{HC}$ and NAD $(\mathrm{F}(2,18)=0.35 ; \quad P=0.71)$, while after $\mathrm{THC}$ we found a significantly lower reward response in NAcc in NAD compared with $\mathrm{HC}(\mathrm{F}(2,18)=7.64 ; P \leqslant 0.001)$. This different effect of THC in HC and NAD proved to be significant as indicated by the main hypothesis test for drug by reward by group interaction $(\mathrm{F}(2,18)=3.73 ; P=0.04)$, indicating that $\mathrm{THC}$ specifically reduced reward anticipation response in NAcc in NAD. The effects were not lateralized as indicated by a nonsignificant $\mathrm{ROI}$ by drug by reward by group interaction $(\mathrm{F}(2,18)=0.11 ; P=0.90)$. There were no significant nonreward-related drug effects in the anticipation phase in NAcc.

Feedback phase. In $\mathrm{HC}$, we found a near significant increase in NAcc response with reward feedback $(\mathrm{F}(1,10)=4.38 ; P=0.06)$. In NAD, we did not find an effect of reward feedback $(F(1,9)=0.05 ; P=0.83)$. However, both after placebo and after THC, there was no difference in reward feedback activity between $\mathrm{HC}$ and NAD (placebo:
$\mathrm{F}(1,19)=0.85 ; P=0.37 ; \mathrm{THC}: \mathrm{F}(1,19)=1.01 ; P=0.33)$. As a result, there was also no difference between the groups in effect of THC on feedback activity in NAcc, as indicated by the non-significant drug by reward by group interaction $(\mathrm{F}(1,19)=0.01 ; P=0.99)$. Also, there was no effect of hemisphere for this interaction $(\mathrm{F}(1,19)=0.13 ; P=0.88)$.

In addition, there were no significant non-reward-related drug effects in the feedback phase in NAcc.

fMRI: caudate putamen. A graphical presentation of the results is shown in Figures $4 a-d$, while statistical tests are shown in Table 3. An illustration of the activity is presented in Figure 5.

Anticipation phase. HC showed an increase in CPU brain activity with increasing reward $(F(2,9)=11.6 ; \quad P<0.001)$. In NAD, we did not find a significant increase in CPU activity with reward $(F(2,8)=1.03 ; P=0.40)$. In the placebo session, there was no difference in effect of reward on brain activity between $\mathrm{HC}$ and $\operatorname{NAD}(\mathrm{F}(2,18)=1.89 ; P=0.18)$. In the $\mathrm{THC}$ session, there was a significantly smaller effect of reward in $\mathrm{NAD}$ than in $\mathrm{HC}(\mathrm{F}(2,18)=4.36 ; P=0.03)$. There was, however, no significant difference between the groups in effect of THC on feedback activity in NAcc, as indicated by the nonsignificant drug by reward by group interaction $(F(2.18)=2.50$; $P=0.11)$. There was also no difference between hemispheres, as indicated by a non-significant hemisphere effect (hemisphere by drug by reward by group: $\mathrm{F}(2,18)=1.41 ; P=0.27)$. 

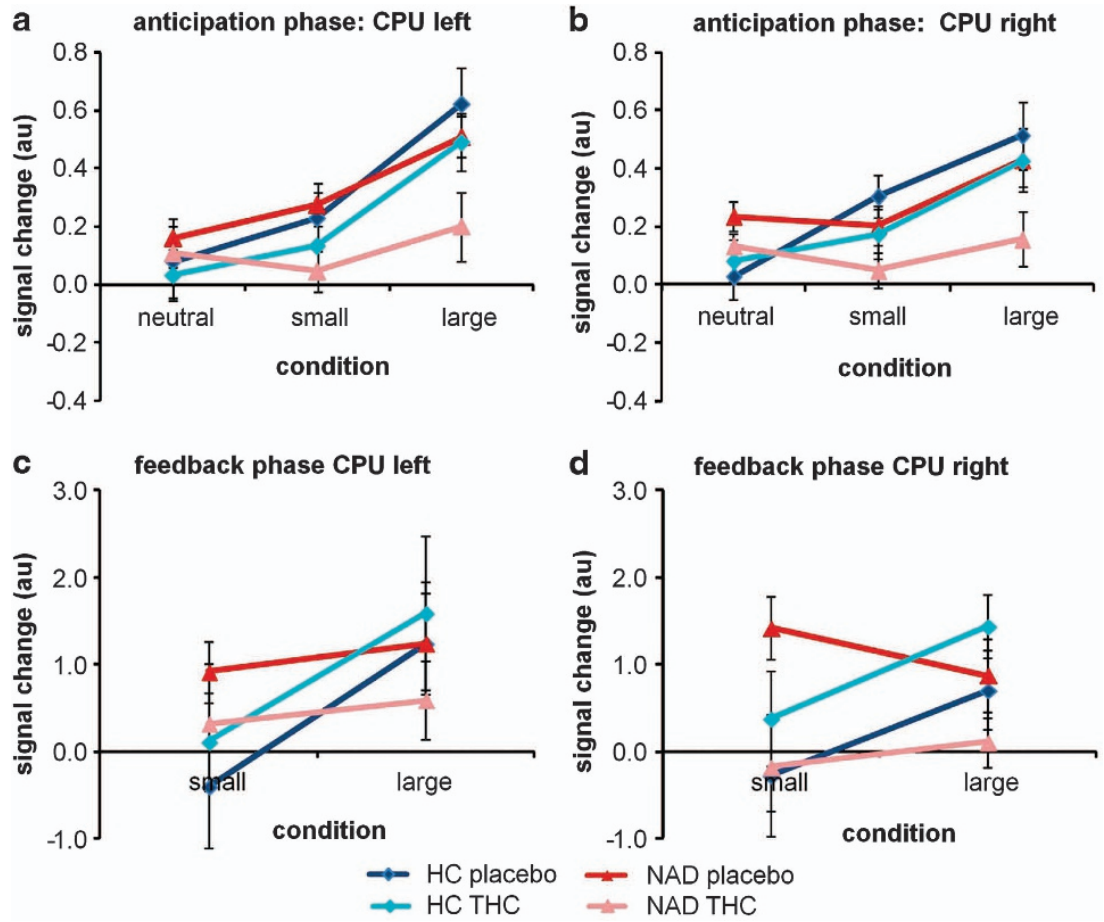

Figure 4 Above: reward anticipation activity in the (a) left and (b) right caudate putamen (CPU) for the conditions (neutral', 'small' or 'large'); (blue: healthy controls (HC); red: subjects with a nicotine addiction (NAD); dark: placebo; light: $\Delta^{9}$-tetrahydrocannabinol (THC)). Over hemispheres, HC showed a significant increase in activity with increasing reward. NAD did not show a significant increase, but group differences were not significant. Below: reward feedback activity in the (c) left and (d) right CPU for the feedback conditions with a reward ('small' or 'large'); (blue: HC; red: NAD; dark: placebo; light: THC). HC showed a significant increase with increasing reward, while NAD did not. However, group differences were not significant. (au, arbitrary units)

Other non-reward-related effects in CPU indicated that NAD showed a general reduction in activity in anticipation phase after THC that was near significant $(\mathrm{F}(1,9)=4.74 ; P=0.06)$, while $\mathrm{HC}$ did not $(\mathrm{F}=0.30 ; P=0.60)$. There was, however, no significant difference between the groups $(F(1,19)=0.85$; $P=0.37)$.

Feedback phase. In the feedback phase, HC showed a significant increase in CPU activity with increasing reward $(F(1,10)=6.76 ; P=0.03)$. This increase was not present in NAD $(F(1,9)=0.01 ; P=0.99)$. However, both in the placebo session as well as in the THC session, we did not find a group difference in brain activity in CPU related to feedback (placebo: $\mathrm{F}(1,19)=1.81 ; P=0.19$; THC: $\mathrm{F}=1.16 ; P=0.30)$. Also, there was no difference in the effect of $\mathrm{THC}$ on reward feedback-related activity between $\mathrm{HC}$ and NAD $(F(1,19)=0.07 ; 0.80)$. Additionally, there were no significant reward-independent feedback effects in CPU.

Aversive trials. Although the aversive trials included in our design were only meant to increase response on rewarding trials and not meant to be included in the analysis, for completeness we report that a separate analysis of these trials showed that for placebo the amplitude for aversive trials was in between the small and larger reward. As in the reward trials, NAD also showed a significantly reduced NAcc response for reward anticipation after THC in the aversive trials. However, group effects were not significant.

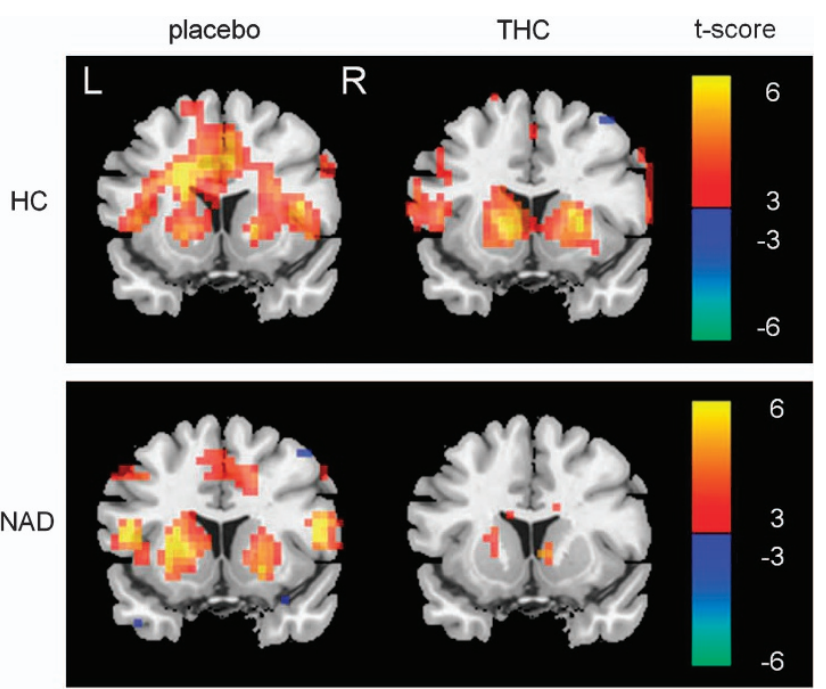

Figure 5 Illustration of brain activity during anticipation (coronal slices at MNI (Montreal Neurological Institute) y-coordinate 12, contrast: large versus rest, $|t|>3.0$, L, left hemisphere, $R$, right hemisphere). Above: healthy controls $(\mathrm{HC})$, below: subjects with a nicotine addiction (NAD). Left: placebo session, right: $\Delta^{9}$-tetrahydrocannabinol (THC) session.

\section{Discussion}

The present study tested the hypothesis that nicotine addiction is associated with abnormal involvement of the eCB system in reward processing. This hypothesis was tested using an eCB challenge with THC in a pharmacological fMRI 
experiment. Subjective measures indicated that THC had a measurable effect on the mental state of our subjects. Both $\mathrm{HC}$ and NAD reported increased levels of 'feeling high', reduced alertness, reduced contentedness and external perception. Calmness was only significantly reduced in $\mathrm{HC}$, but the level of the reduction was small.

We measured the functional response in NAcc and CPU to anticipation and feedback of monetary reward after placebo and $\mathrm{THC}$ in both of the groups. The key finding of the study was that $\mathrm{HC}$ and NAD showed opposite effects in NAcc response on monetary reward anticipation after THC administration. Although THC administration in NAD caused a significantly reduced NAcc response, in HC there was a slightly increased NAcc response. The effects appear to be similar but weaker in the CPU. First, there was also no significant difference in reward anticipation response in the placebo session. Second, we also found a significant lower anticipation response in NAD than in $\mathrm{HC}$ in the THC session. However, in CPU, unlike in NAcc, the difference between the placebo and the THC session was not significant.

These results support the notion that altered eCB dynamics in the context of reward processing have a role in nicotine addiction, adding human findings to the evidence that the eCB system is involved in nicotine addiction. ${ }^{37}$ Our expectation that $\mathrm{THC}$ would have an opposite effect on reward processing in $\mathrm{HC}$ and NAD appeared to be supported by the reward anticipation results in NAcc. As can be seen in Figures $3 a$ and $b, T H C$ reduces brain activity in NAcc in NAD, whereas this effect appears to be opposite in $\mathrm{HC}$.

Our results suggest that in nicotine addiction the eCB system may have a stronger influence on the reward system than in $\mathrm{HC}$. In $\mathrm{HC}$, the slight increase in reward anticipation activity (although by itself not significant) is in line with previous reports that indicate that administration of eCB antagonist Rimonabant can reduce rewarding effects. ${ }^{17}$ By contrast, the absence of an anticipatory NAcc response to monetary reward after THC in NAD suggests the possibility of a ceiling effect on activity in NAcc, which is not present in $\mathrm{HC}$. If activity is strongly elevated by THC in NAD during baseline, this may leave less room for further elevation of activity by the monetary stimuli. This difference between HC and NAD could, therefore, be due to a maximized level of dopamine receptor occupation in NAD after THC due to reduced availability of dopamine D2 receptors. ${ }^{38}$ In $\mathrm{HC}, \mathrm{THC}$ also increases dopamine receptor occupancy, but as dopamine receptor availability is not compromised, there is room for a further increase in response to a monetary reward. In addition, it is also possible that nicotine addiction is associated with altered sensitivity to THC or non-dopaminergic mechanisms in NAcC (for example, Berridge and Kringelbach ${ }^{28}$ ).

The abnormal NAcc response in NAD to monetary rewards after THC may be related to prodromal differences in eCB function, potentially constituting a predisposition to addiction. Activation of nicotinic receptors on dopaminergic neurons in the ventral tegmental area by nicotine is hypothesized to increase release of the eCB AEA ${ }^{39,40}$ Functional mutations in the $C B 1$ cannabinoid receptor and fatty acid amide hydrolyze genes (FAAH; the main enzyme that breaks down AEA) have been linked to rewarding effects of nicotine as well as other drugs such as cocaine, alcohol, marijuana and heroin. ${ }^{10,37,41,42}$ Inhibition of FAAH with a selective inhibitor URB 597 has been shown to enhance nicotine reward in mice. ${ }^{37}$ This is hypothesized to be a direct result of increased levels of AEA. On the other hand, URB 597 has also been shown to neutralize rewarding effects of nicotine. ${ }^{43-45}$ The proposed mechanisms for this effect is that AEA is protected from rapid degradation by URB597, resulting in increased activation of $\mathrm{CB} 1$ receptors. This reduces activation of dopaminergic neurons and release of dopamine from their terminals in the NAcc shell. It has been previously suggested that the different effects are dose related. ${ }^{46}$ Although the exact working mechanisms may still be unknown, a recent review provides several convincing arguments for a direct link between the eCB system and addiction, associated with polymorphisms in the $\mathrm{CB} 1$ cannabinoid receptor and $\mathrm{FAAH}$ genes. ${ }^{7}$ The current study is one of the first to add human brain imaging results to support this view.

Although the present study sample only included subjects with a nicotine addiction, the results may be relevant for other drug addictions as well. It is known that addiction to nicotine causes comparable reductions in striatal DA D2 receptor density as addiction to other drugs of abuse. ${ }^{38,47}$ There is evidence suggesting an interaction between the eCB system and alcohol dependence. ${ }^{48}$ In addition, animal studies have shown that chronic exposure to nicotine, alcohol and THC (but not cocaine) increase eCB levels in the limbic forebrain, including the NAcc. ${ }^{49,50}$ These results suggest the possibility of a general involvement of eCB in drug addiction. ${ }^{2}$ However, whether our results can be extrapolated to addiction in general warrants further investigation.

In addition to the main finding, there were several other notable findings in this study.

Our results in the placebo session in HC support those previously found with the MID task, as previous studies using this paradigm have indicated that anticipation of an increasing reward evokes an increasing response that is most pronounced in NAcc. ${ }^{24,51}$

Our results also showed feedback-related activity in $\mathrm{HC}$ in CPU that was reward-size dependent. However, in contrast to previous studies using the MID paradigm, we also found a near significant reward feedback activity in NAcc. ${ }^{24,52}$ This finding is potentially related to the applied ROI-based analysis, which is more powerful than the more commonly applied voxel-wise analysis.

In contrast to some previous studies, we did not find a significant difference between $\mathrm{HC}$ and NAD in reward anticipation activity in NAcc after placebo. What may have influenced the NAcc results is the fact that all our subjects were occasional cannabis users, as well as that our $\mathrm{HC}$ were occasional smokers. Indeed, in a previous study, we found that NAD as well as cannabis users both showed a significantly reduced reward anticipation response in NAcc, compared with subjects who had never smoked or used cannabis. ${ }^{15}$ It has to be noted, however, that previous results in this regard are inconsistent, as some have reported reduced response to monetary reward anticipation in nicotine addiction, ${ }^{22,23}$ while others did not. ${ }^{21,53}$ Likewise, reward studies using alcoholdependent subjects also provide inconsistent results. Two studies have reported decreased activation in ventral 
striatum, ${ }^{54,55}$ and one study showed normal activity during reward anticipation in the MID task. ${ }^{56}$

In our study, NAD showed reduced feedback response in CPU, (and a trend for a similar effect in NAcc) after THC. This is also in line with the presented hypothesis that THC maximally activated the reward system in NAD, leaving less room for an additional response to monetary reward.

Behaviorally, we found that THC increased RT in HC as well as NAD. However, this effect was not reward dependent. The general effect of THC on RT is consistent with the reduced perceived alertness, measured with the VAS. Also, we did not find any group differences in the effect of THC on performance despite the difference in brain activity. There can be several explanations for this. The general slowing effect of $\mathrm{THC}$, indicated by a significant main effect of drug on performance, may have introduced too much betweensubject variation. Also, it is possible that reward-specific effects of THC are present but are too small to become significant owing to our relatively small sample. The RT effects do, however, appear to go in the same direction that can be expected based on the activity results, as the increase in RT in the largest reward condition is larger for NAD then for $\mathrm{HC}$, as can be seen in Figure 2.

Somewhat surprisingly, we did not find an effect of THC on reward anticipation reactivity in CPU and NAcc in $\mathrm{HC}$. Human imaging studies on these effects are sparse, and to our knowledge there is only one other study on this topic, also from our group. ${ }^{16}$ In that study, using a comparable experimental design, we also did not find a significant effect of THC on NAcc activity. It has to be noted, however, that NAcc showed low activity for all conditions in that study. In addition, Van Hell et al. ${ }^{16}$ did report significant striatal response to reward anticipation that also was not affected by THC. As the current study replicated this finding, the lack of effect of THC on reward anticipation in humans (not addicted to nicotine) appears to be a reliable finding. It has to be taken into account, however, that due to ethics protocol requirements, in both studies $\mathrm{HC}$ were infrequent nicotine users as well as infrequent cannabis users, as THC is typically mixed with tobacco. This in contrast to several other studies where $\mathrm{HC}$ were nicotine-free. ${ }^{15,22,53,57}$ This also may have reduced the difference between NAD and $\mathrm{HC}$ in the placebo session.

Our results may also have been influenced by the fact that NAD were nicotine-abstinent for about $3 \mathrm{~h}$ when performing the experimental task. This period was needed to ensure absence of acute effects of nicotine. The possibility that subjects were experiencing some withdrawal effects at the time of testing can, however, not be excluded, as early effects of withdrawal have been reported within 60 min. ${ }^{58}$ However, responses to the VAS during scanning did not suggest effects of withdrawal in NAD.

Although our $\mathrm{ROI}$ analyses are not corrected for multiple comparison, we do not feel that we capitalized on chance, as we only tested two ROls. Additionally, although the main three-way interaction in NAcc changes to near significance after correction for two ROIs ( $P=0.06)$, many of the follow-up ANOVA effects that drive this interaction do survive correction for two ROls. In this study, a cannabinoid agonist was used to investigate the role of the endogenous cannabinoid system. Although this is a well-established method of studying cannabinoid function, a disadvantage is that cannabinoid agonists like THC can activate cannabinoid receptors everywhere in the brain, regardless of the endogenous activity of the system. Future experiments that make use of a cannabinoid antagonist could have an important role in confirming the role of the endogenous system in reward processing in addiction.

We did not test for differences between HC and NAD in impulsivity, anxiety and depression. Also, there was a trend that the average age of NAD was higher than $\mathrm{HC}$ in the current study. These variables may have influenced our results. However, it should be noted that potential group differences were not reflected in the VAS, except for a small effect in 'calmness', but this showed that in $\mathrm{HC}$ calmness was more reduced after THC. Also, target presentation time, which was based on the shortest RT in a task practice session, was not significantly different between $\mathrm{HC}$ and NAD, indicating that a potential group difference in impulsivity did not affect task performance.

As THC was dissolved in $100 \mathrm{vol} \%$ alcohol and the solvent was used as placebo, we cannot exclude that the inhalation of alcohol might have affected blood alcohol concentration. However, as only $600 \mu \mathrm{l}$ alcohol was administered, this is very unlikely. As a comparison, this amount is less than one-tenth of one alcoholic beverage.

In conclusion, our study showed that in NAD the NAcc response to reward anticipation is significantly reduced after THC administration, while the response in $\mathrm{HC}$ was the opposite. These results suggest that nicotine addiction is associated with altered eCB modulation of reward processing in the NAcc. This study adds human findings to the existing evidence that identifies the eCB system as a therapeutic candidate for nicotine addiction.

\section{Conflict of interest}

The authors declare no conflict of interest.

Acknowledgements. The PhICS study is performed within the framework of Top Institute Pharma, project number T5-107. This study is registered in the EudraCT database (2007-004247-30) and the Dutch Trial Register (NTR1787). We would like to thank Storz and Bickel for kindly supplying the Volcano vaporizer, and Annelies Brouwer, Erik Oudman, Joep van der Graaf, David Terburg and Estrella Montoya for help with data acquisition and analysis.

1. EMCDDA. The state of the drugs problem in Europe 2010.

2. Maldonado R, Valverde O, Berrendero F. Involvement of the endocannabinoid system in drug addiction. Trends Neurosci 2006; 29: 225-232.

3. Castañé A, Berrendero $F$, Maldonado R. The role of the cannabinoid system in nicotine addiction. Pharmacol Biochem Behav 2005; 81: 381-386.

4. Le Foll B, Forget B, Aubin HJ, Goldberg SR. Blocking cannabinoid CB1 receptors for the treatment of nicotine dependence: insights from pre-clinical and clinical studies. Addict Biol 2008; 13: 239-252.

5. Valjent E, Mitchell JM, Besson M-J, Caboche J, Maldonado R. Behavioural and biochemical evidence for interactions between Delta 9-tetrahydrocannabinol and nicotine. Br J Pharmacol 2002; 135: 564-578

6. Scherma M, Fadda P, Le Foll B, Forget B, Fratta W, Goldberg SR et al. The endocannabinoid system: a new molecular target for the treatment of tobacco addiction. CNS Neurol Disord Drug Targets 2008; 7: 468-481.

7. Lopez-Moreno JA, Echeverry-Alzate V, Buhler KM. The genetic basis of the endocannabinoid system and drug addiction in humans. J Psychopharmacol 2012; 26: 133-143.

8. Cohen C, Perrault G, Voltz C, Steinberg R, Soubrie P. SR141716, a central cannabinoid $(\mathrm{CB}(1))$ receptor antagonist, blocks the motivational and dopamine-releasing effects of nicotine in rats. Behav Pharmacol 2002; 13: 451-463. 
9. De Vries TJ, Schoffelmeer AN. Cannabinoid CB1 receptors control conditioned drug seeking. Trends Pharmacol Sci 2005; 26: 420-426.

10. Cippitelli A, Astarita G, Duranti A, Caprioli G, Ubaldi M, Stopponi S et al. Endocannabinoid regulation of acute and protracted nicotine withdrawal: effect of FAAH inhibition. PIOS ONE 2011; 6: e28142

11. Cahill K, Ussher M. Cannabinoid type 1 receptor antagonists (rimonabant) for smoking cessation. Cochrane Database Syst Rev 2007; 17: CD005353.

12. van der Stelt M, Di Marzo V. The endocannabinoid system in the basal ganglia and in the mesolimbic reward system: implications for neurological and psychiatric disorders. Eur J Pharmacol 2003; 480: 133-150.

13. Solinas M, Goldberg SR, Piomelli D. The endocannabinoid system in brain reward processes. Br J Pharmacol 2008; 154: 369-383.

14. Haber SN, Knutson B. The reward circuit: linking primate anatomy and human imaging. Neuropsychopharmacology 2010; 35: 4-26.

15. van Hell HH, Vink M, Ossewaarde L, Jager G, Kahn RS, Ramsey NF. Chronic effects of cannabis use on the human reward system: an fMRI study. Eur Neuropsychopharmacol 2010; 20: 153-163.

16. van Hell HH, Jager G, Bossong MG, Brouwer A, Jansma JM, Zuurman L et al. Involvement of the endocannabinoid system in reward processing in the human brain. Psychopharmacology (Berl) 2012; 219: 981-990.

17. Horder J, Harmer CJ, Cowen PJ, McCabe C. Reduced neural response to reward following 7 days treatment with the cannabinoid CB1 antagonist rimonabant in healthy volunteers. Int J Neuropsychopharmacol 2010; 13: 1103-1113.

18. Curtis VA, Bullmore ET, Brammer MJ, Wright IC, Williams SC, Morris RG et al. Attenuated frontal activation during a verbal fluency task in patients with schizophrenia Am J Psychiatry 1998; 155: 1056-1063.

19. Williams CM, Kirkham TC. Observational analysis of feeding induced by Delta9-THC and anandamide. Physiol Behav 2002; 76: 241-250.

20. Martin-Soelch C, Leenders KL, Chevalley AF, Missimer J, Kunig G, Magyar S et al. Reward mechanisms in the brain and their role in dependence: evidence from neurophysiological and neuroimaging studies. Brain Res Brain Res Rev 2001; 36: 139-149.

21. Hommer DW, Bjork JM, Gilman JM. Imaging brain response to reward in addictive disorders. Ann N Y Acad Sci 2011; 1216: 50-61.

22. Rose EJ, Ross TJ, Salmeron BJ, Lee M, Shakleya DM, Huestis M et al. Chronic exposure to nicotine is associated with reduced reward-related activity in the striatum but not the midbrain. Biol Psychiatry 2012; 71: 206-213.

23. Bühler M, Vollstädt-Klein S, Kobiella A, Budde H, Reed LJ, Braus DF et al. Nicotine dependence is characterized by disordered reward processing in a network driving motivation. Biol Psychiatry 2010; 67: 745-752.

24. Knutson B, Adams CM, Fong GW, Hommer D. Anticipation of increasing monetary reward selectively recruits nucleus accumbens. J Neurosci 2001; 21: RC159.

25. Everitt BJ, Robbins TW. Neural systems of reinforcement for drug addiction: from actions to habits to compulsion. Nat Neurosci 2005; 8: 1481-1489.

26. Kalivas PW, O'Brien C. Drug addiction as a pathology of staged neuroplasticity. Neuropsychopharmacology 2008; 33: 166-180.

27. Koob GF, Volkow ND. Neurocircuitry of addiction. Neuropsychopharmacology 2010; 35 : 217-238.

28. Berridge KC, Kringelbach ML. Affective neuroscience of pleasure: reward in humans and animals. Psychopharmacology (Berl) 2008; 199: 457-480.

29. van Hell HH, Bossong MG, Jager G, Kahn RS, Ramsey NF. Methods of the pharmacological imaging of the cannabinoid system (PhICS) study: towards understanding the role of the brain endocannabinoid system in human cognition. Int $J$ Methods Psychiatr Res 2011; 20: 10-27.

30. van Vliet IM, Leroy H, Van Megen HGJM. M.I.N.I. Internationaal Neuropsychiatrisch Interview. Nederlandse Versie 5.0.02000.

31. Heatherton TF, Kozlowski LT, Frecker RC, Fagerstrom KO. The Fagerstrom Test for Nicotine Dependence: a revision of the Fagerstrom Tolerance Questionnaire. Br J Addict 1991; 86: 1119-1127.

32. Bond $A$, Lader $M$. The use of analogue scales in rating subjective feelings. $\mathrm{Br} J \mathrm{Med}$ Psychol 1974; 47 : 7 .

33. Bowdle TA, Radant AD, Cowley DS, Kharasch ED, Strassman RJ, Roy-Byrne PP. Psychedelic effects of ketamine in healthy volunteers: relationship to steady-state plasma concentrations. Anesthesiology 1998; 88: 6 .

34. Zuurman L, Roy C, Schoemaker RC, Hazekamp A, den Hartigh J, Bender JC et al. Effect of intrapulmonary tetrahydrocannabinol administration in humans. J Psychopharmacol 2008; 22: 707-716.

35. Neggers SF, Hermans EJ, Ramsey NF. Enhanced sensitivity with fast three-dimensional blood-oxygen-level-dependent functional MRI: comparison of SENSE-PRESTO and 2D-EPI at 3T. NMR Biomed 2008; 21: 663-676

36. Tzourio-Mazoyer N, Landeau B, Papathanassiou D, Crivello F, Etard O, Delcroix N et al. Automated anatomical labeling of activations in SPM using a macroscopic anatomical parcellation of the MNI MRI single-subject brain. Neuroimage 2002; 15: 273-289.

37. Merritt LL, Martin BR, Walters C, Lichtman AH, Damaj Ml. The endogenous cannabinoid system modulates nicotine reward and dependence. J Pharmacol Exp Ther 2008; 326: 483-492.

38. Fehr C, Yakushev I, Hohmann N, Buchholz HG, Landvogt C, Deckers $\mathrm{H}$ et al. Association of low striatal dopamine $\mathrm{d} 2$ receptor availability with nicotine dependence similar to that seen with other drugs of abuse. AM J Psychiatry 2008; 165: 507-514.

39. Di Marzo V, Fontana A, Cadas H, Schinelli S, Cimino G, Schwartz JC et al. Formation and inactivation of endogenous cannabinoid anandamide in central neurons. Nature 1994; 372: 686-691.

40. Melis M, Pistis M, Perra S, Muntoni AL, Pillolla G, Gessa GL. Endocannabinoids mediate presynaptic inhibition of glutamatergic transmission in rat ventral tegmental area dopamine neurons through activation of CB1 receptors. J Neurosci 2004; 24: 53-62.

41. Orio L, Crespo I, Lopez-Moreno JA, Reyes-Cabello C, Rodriguez de FonsecaF, Gomez de HerasR. Additive effects of cannabinoid CB1 receptors blockade and cholecystokinin on feeding inhibition. Pharmacol Biochem Behav 2011; 98: 220-226.

42. Castane A, Valjent E, Ledent C, Parmentier M, Maldonado R, Valverde O. Lack of CB1 cannabinoid receptors modifies nicotine behavioural responses, but not nicotine abstinence. Neuropharmacology 2002; 43: 857-867.

43. Scherma M, Panlilio LV, Fadda P, Fattore L, Gamaleddin I, Le Foll B et al. Inhibition of anandamide hydrolysis by cyclohexyl carbamic acid 3'-carbamoyl-3-yl ester (URB597) reverses abuse-related behavioral and neurochemical effects of nicotine in rats. J Pharmacol Exp Ther 2008; 327: 482-490.

44. Fales CL, Barch DM, Rundle MM, Mintun Ma, Snyder AZ, Cohen JD et al. Altered emotional interference processing in affective and cognitive-control brain circuitry in major depression. Biol Psychiatry 2008; 63: 377-384.

45. Forget B, Coen KM, Le Foll B. Inhibition of fatty acid amide hydrolase reduces reinstatement of nicotine seeking but not break point for nicotine self-administration-comparison with $\mathrm{CB}$ (1) receptor blockade. Psychopharmacology (Berl) 2009; 205: 613-624.

46. Scherma M, Medalie J, Fratta W, Vadivel SK, Makriyannis A, Piomelli D et al. The endogenous cannabinoid anandamide has effects on motivation and anxiety that are revealed by fatty acid amide hydrolase (FAAH) inhibition. Neuropharmacology 2008; 54 $129-140$.

47. Volkow ND, Fowler JS, Wang GJ, Baler R, Telang F. Imaging dopamine's role in drug abuse and addiction. Neuropharmacology 2009; 56(Suppl 1): 3-8.

48. Pava MJ, Woodward JJ. A review of the interactions between alcohol and the endocannabinoid system: implications for alcohol dependence and future directions for research. Alcohol 2012; 46: 185-204.

49. Di Marzo V, Berrendero F, Bisogno T, Gonzalez S, Cavaliere P, Romero J et al. Enhancement of anandamide formation in the limbic forebrain and reduction of endocannabinoid contents in the striatum of delta9-tetrahydrocannabinol-tolerant rats. J Neurochem 2000; 74: 1627-1635.

50. Gonzalez S, Cascio MG, Fernandez-Ruiz J, Fezza F, Di Marzo V, Ramos JA. Changes in endocannabinoid contents in the brain of rats chronically exposed to nicotine, ethanol or cocaine. Brain Res 2002; 954: 73-81.

51. Knutson B, Fong GW, Bennett SM, Adams CM, Hommer D. A region of mesial prefrontal cortex tracks monetarily rewarding outcomes: characterization with rapid event-related fMRI. Neuroimage 2003; 18: 263-272.

52. O'Doherty JP, Deichmann R, Critchley HD, Dolan RJ. Neural responses during anticipation of a primary taste reward. Neuron 2002; 33: 815-826.

53. Martin-Soelch C, Leenders KL, Chevalley aF, Missimer J, Künig G, Magyar S et al. Reward mechanisms in the brain and their role in dependence: evidence from neurophysiological and neuroimaging studies. Brain Res Rev 2001; 36: 139-149.

54. Beck A, Schlagenhauf F, Wustenberg T, Hein J, Kienast T, Kahnt T et al. Ventral striatal activation during reward anticipation correlates with impulsivity in alcoholics. Biol Psychiatry 2009; 66: 734-742.

55. Wrase J, Schlagenhauf F, Kienast T, Wustenberg T, Bermpohl F, Kahnt T et al. Dysfunction of reward processing correlates with alcohol craving in detoxified alcoholics. Neuroimage 2007; 35: 787-794.

56. Bjork JM, Smith AR, Hommer DW. Striatal sensitivity to reward deliveries and omissions in substance dependent patients. Neuroimage 2008; 42: 1609-1621.

57. Martin-Soelch C, Missimer J, Leenders KL, Schultz W. Neural activity related to the processing of increasing monetary reward in smokers and nonsmokers. Eur $\mathrm{J}$ Neurosci 2003; 18: 680-688.

58. Hendricks PS, Ditre JW, Drobes DJ, Brandon TH. The early time course of smoking withdrawal effects. Psychopharmacology (Berl) 2006; 187: 385-396.

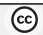

SOMERIEHTS

licensed under the Creative Commons Attribution-NonCommercial-No Derivative Works 3.0 Unported License. To view a copy of this license, visit http://creativecommons.org/licenses/by-nc-nd/3.0/ 